\title{
A high-resolution TOF detector - a possible way to compete with a RICH detector."
}

\author{
J. Va'vra", C. Ertley ${ }^{+}$, D.W.G.S. Leith, B. Ratcliff, and J. Schwiening \\ SLAC, Stanford University, CA 94309, U.S.A." \\ Argonne National Laboratory, Argonne, Il 60439, U.S.A. ${ }^{+}$
}

To be published in Nuclear Instr. \& Methods.

\begin{abstract}
Using two identical 64-pixel Burle/Photonis MCP-PMTs to provide start and stop signals, we have achieved a timing resolution of $\sigma_{\text {Single_detector }} \sim 7.2 \mathrm{ps}$ for $\mathrm{N}_{\mathrm{pe}} \sim 100$ photoelectrons $\left(\mathrm{N}_{\mathrm{pe}}\right)$ with a laser diode providing a $1 \mathrm{~mm} \mathrm{spot}$ on the MCP window. The limiting resolution achieved was $\sigma_{\text {Single_detector }} \sim 5.0 \mathrm{ps}$ for $\mathrm{N}_{\mathrm{pe}} \sim 250$, for which we estimate the MCP-PMT contribution of $\sigma_{M C P-P M T} \sim 4.5 \mathrm{ps}$. The electronics contribution is estimated as $\sigma_{\text {Electronics }}=3.42 \mathrm{ps}$. These results suggest that an ultra-high resolution TOF detector may become a reality at future experiments one day.
\end{abstract}

Keywors: Photodetectors, Cherenkov detectors, RICH, TOF

\section{Introduction}

We present new timing measurements with Burle/Photonis MCP-PMT (micro-channel plate PMT) with $10 \mu \mathrm{m}$ holes. Our measurements use two identical MCP-PMT and electronics setups, driven from a fiber splitter and operated in a relative start-stop mode (which eliminates drifts in an external laser diode start signal). A similar arrangement was used in the test beam. We have operated both MCP-PMTs at a lower gain $\left(<10^{5}\right)$, where the detection is not sensitive to single photoelectrons, but where it has a linear response in the range of $\mathrm{N}_{\mathrm{pe}} \sim 30-50$ photoelectrons. This is a departure from the previous methodology [1], where we operated in single photoelectron mode. This provided good timing resolution already at $\mathrm{N}_{\mathrm{pe}} \sim 20$, however, at the expense of worse linearity, aging, rate handling capability, and pulse recovery.

- Manuscript received on January 2, 2008.

\# Corresponding author. tel.: 650-926-2658; fax: 650-926-4178; e-mail: jjv@ slac.stanford.edu.

** Work supported by the Department of Energy, contract DEAC02-76SF00515. 
(a)

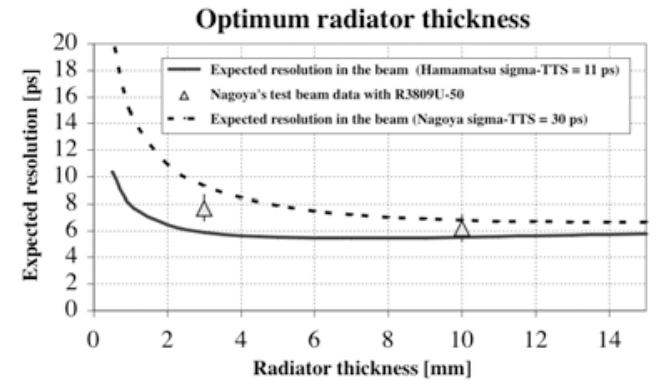

(b)

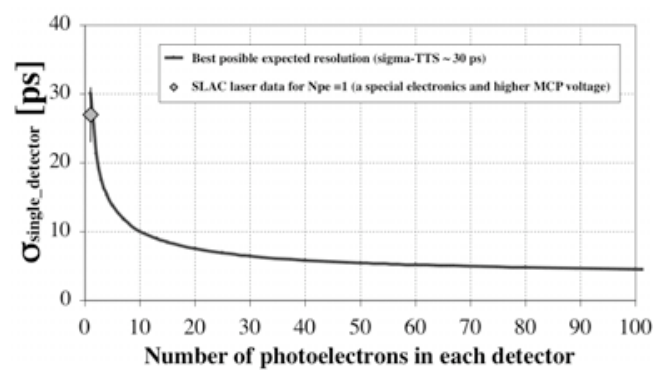

Fig. 1. Expected resolution with a simple model (a) in a beam ${ }^{1}$ as a function of radiator thickness, and (b) with a laser ${ }^{2}$ as a function of number of photoelectrons. The calculation assumes measured numbers of $\sigma_{\mathrm{TTS}} \sim 27 \mathrm{ps}$ for our laser test [1], and $\sigma_{\text {TTS }} \sim 11 \mathrm{ps}^{3}$ or $\sigma_{\mathrm{TTS}} \sim 30 \mathrm{ps}^{4}$ for Nagoya beam test [2].

Fig. 1 shows the expected resolution in the beam and with a laser. These are simple estimates, which neglect intricacies of pulse shaping, constant-fraction (CFD) timing, photoelectron creation and transport, MCP multiplication, etc.

The presented TOF detector is being considered as one possible option for a Super-B PID detector [3] in the forward/backward regions. For that application, all 64 pads need to be instrumented, the detector must work at 16 $\mathrm{kG}$, its radiator would be a 7-10 mm thick MCP-PMT's window, and its electronics would be based either on a similar concept as presented, or a waveform digitization.

Generally, a TOF-based PID is competitive with a RICH PID, if one can obtain $\sigma_{\mathrm{TOF}} \sim 5-10 \mathrm{ps}$ (for an Aerogel RICH with $\mathrm{n} \sim 1.03$ ), or $\sigma_{\mathrm{TOF}} \sim 15-20$ ps (for a DIRC-like RICH with $\mathrm{n} \sim 1.47$ ), and one has at least $2 \mathrm{~m}$ of TOF path [3]. There is no practical way to compete with a gaseous RICH at higher momenta [4].

\section{Experimental setup.}

Fig. 2 shows the MCP-PMT enclosure with a Fused silica radiator $(10 \mathrm{~mm}$ dia., $10 \mathrm{~mm}$ long) and the fiber optics. MCP-PMT has 64 pads; four pads under the radiator were shorted together and connected to an amplifier. The rest of the pads were shorted to ground. The laser diode optics produced a $1 \mathrm{~mm}$ spot on the MCP face, while the ESA test beam had a spot size of $\sigma \sim 1-2 \mathrm{~mm}$. Two identical MCP-PMT detectors were prepared, both having 10 $\mu \mathrm{m}$ dia. holes ${ }^{5}$. Electronics ${ }^{6}$ of this test and its pulser ${ }^{7}$ calibration are shown on Fig. 3. The laser light was split in a fiber splitter.

\footnotetext{
${ }^{1}$ Beam: $\sigma \sim \sqrt{ }\left[\sigma_{\text {MCP-PMT }}^{2}+\sigma_{\text {Radiator }}^{2}+\sigma_{\text {Pad }}^{2}+\sigma_{\text {Electronics }}^{2}\right] \sim \sqrt{ }\left[\left(\sigma_{\mathrm{TTS}} / \sqrt{ } \mathrm{N}_{\mathrm{pe}}\right)^{2}+\left(\left(\left(\mathrm{L} / \mathrm{cos} \Theta_{\mathrm{C}}\right) /(300 \mathrm{~mm} / \mathrm{ps}) / \mathrm{n}_{\text {group }}\right) / \sqrt{ }(12 \mathrm{Npe})\right)^{2}+\left(\left(\mathrm{L}_{\mathrm{pad}}\right.\right.\right.$ $\left.1300 \mathrm{~mm} / \mathrm{ps}) / \sqrt{ }(12 \mathrm{Npe}))^{2}+(3.42 \mathrm{ps})^{2}\right]$, where $\mathrm{L}$ is a radiator length, $\mathrm{L}_{\mathrm{pad}}$ is a pixel size, $\mathrm{N}_{\mathrm{pe}}$ is a number of photoelectrons, and $\mathrm{n}_{\text {group }}$ is a group refraction index.

${ }^{2}$ Laser: $\left.\sigma \sim \sqrt{ }\left[\sigma_{\text {MCP-PMT }}^{2}+\sigma_{\text {Laser }}^{2}+\sigma_{\text {Electronics }}^{2}\right] \sim \sqrt{ }\left[\sigma_{\text {TTS }} / \sqrt{ } \mathrm{N}_{\text {pe }}\right)^{2}+\sqrt{ }\left((\text { FWHM } / 2.35) / \sqrt{ } \mathrm{N}_{\text {pe }}\right)^{2}+(3.42 \mathrm{ps})^{2}\right] \sim \sqrt{ }\left[3.8^{2}+1.8^{2}+3.42^{2}\right] \sim 5.4$ ps for Npe $=50$ photoelectrons;

PiLas laser diode is made by Advanced Laser Diode Systems, D-12489 Berlin, Germany.

${ }^{3}$ Hamamatsu data sheet info for R3809U-50 tube: $\sigma_{\mathrm{TTS}} \sim 11 \mathrm{ps}$

${ }^{4}$ Nagoya people measured for R3809U-50 tube: $\sigma_{\text {TTS }} \sim 30$ ps.

5 Two Burle/Photonis MCP-PMTs, S/N: $11180401 \& 7300714$

6 Electronics: Ortec TAC 588, CFD 9327, 14 bit ADC 114.

7 200MHz pulser with one start \& multiple equally spaced random stops, made by Impeccable instruments, LLC, Knoxville, Tn., USA, www.ImpeccableInstruments.com.
} 


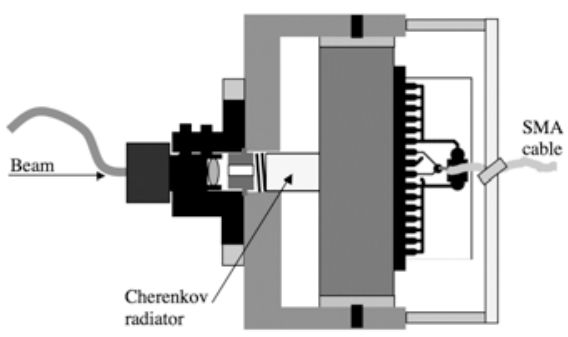

Fig. 2. Two identical detector setups were built to allow a relative start-stop measurement using either a laser or a beam (in the beam we remove the fiber to reduce the mass).

(a)

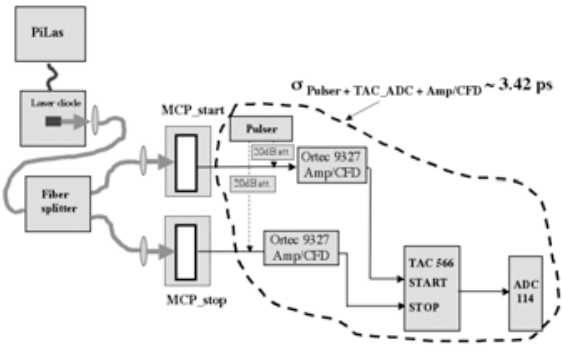

(b)

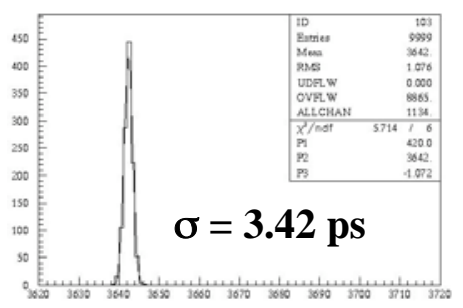

ADC counts

Fig. 3. (a) Electronics ${ }^{6}$ that was used for laser or beam tests, and its calibration scheme (b) Calibration results: $\sigma_{\text {Electronics }}=3.42 \mathrm{ps}$, to which the pulser ${ }^{7}$ contributed $\sigma_{\text {Pulser }}<2$ ps $(3.19 \mathrm{ps} /$ count $)$.

\section{Experimental results with a laser diode}

Fig. 4 shows the time resolution obtained with a laser diode ${ }^{8}$ with a fiber splitter and a tandem of two identical detectors, and each having a signal of $\mathrm{N}_{\mathrm{pe}} \sim 100$ photoelectrons, providing start \& stop TAC signals (the circuit is shown in Fig. 3a). The single detector resolution is obtained by dividing the measured resolution by $\sqrt{2}$, resulting in $\sigma_{\text {Single_detector }}=\sigma / \sqrt{ } 2 \sim 7.2 \mathrm{ps}$. Fig.5 shows this resolution as a function of number of photoelectrons Npe for the CFD arming thresholds of $-10 \mathrm{mV}$, the CFD walk (zero-crossing) threshold of $+5 \mathrm{mV}$ and MCP-PMT voltages of $2.28 \& 2.0 \mathrm{kV}$ respectively, and compares it with a prediction. ${ }^{2}$ This prediction ${ }^{2}$ agrees well with the data if we assume $\sigma_{\text {TTS }} \sim 110 \mathrm{ps}$, which is close to our measured number of $\sigma \sim 106$ ps for Npe $\sim 2-3$. Such a large value of $\sigma_{\text {TTS }}$ is consistent with our choice of being linear for signals of Npe $\sim 30-50$, for which we measure $\sigma \sim 19$ ps, according to Fig. 5.

The limiting resolution at large $\mathrm{N}_{\mathrm{pe}} \sim 250$ (a large number for most of the applications) is found to be $\sigma_{\text {Single_detector }} \sim 5.0 \mathrm{ps}$. We estimate the MCP-PMT contribution to this result is $\sigma$ MCP-PMT $<4.5$ ps. ${ }^{9}$

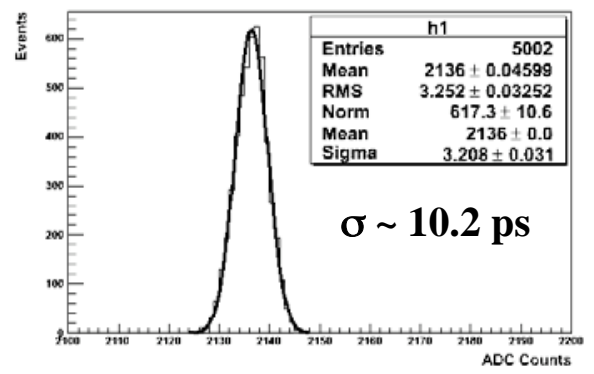

ADC counts

Fig. 4 Time resolution $\left(\mathrm{T}_{\text {start }}-\mathrm{T}_{\text {stop }}\right)$ obtainea witn a laser alode for $\mathrm{Npe} \sim 100$ (3.19 ps/count and $\left.\sigma_{\text {single detector }} \sim \sigma / \sqrt{2} \sim 7.2 \mathrm{ps}\right)$.

\footnotetext{
$\overline{8}$ PiLas laser diode, $635 \mathrm{~nm}$, FWHM light spread $\sim 30 \mathrm{ps}$.

${ }^{9}$ MCP-PMT contribution to resolution: $\sigma_{\text {MCP-PMT }}<\sqrt{ } 1 / 2\left\{\sigma^{2}-\left[\sigma_{\text {Electronics }}^{2}-\sigma_{\text {Pulser }}^{2}\right]\right\}<4.5 \mathrm{ps}$, where $\sigma \sim 7.0 \mathrm{ps}, \sigma_{\text {Pulser }} \sim 2 \mathrm{ps}$, and $\sigma_{\text {Electronics }}=$ $3.42 \mathrm{ps}, \sigma_{\text {Single_detector }}=7.0 \mathrm{ps} / \sqrt{2}=5.0 \mathrm{ps}$.
} 


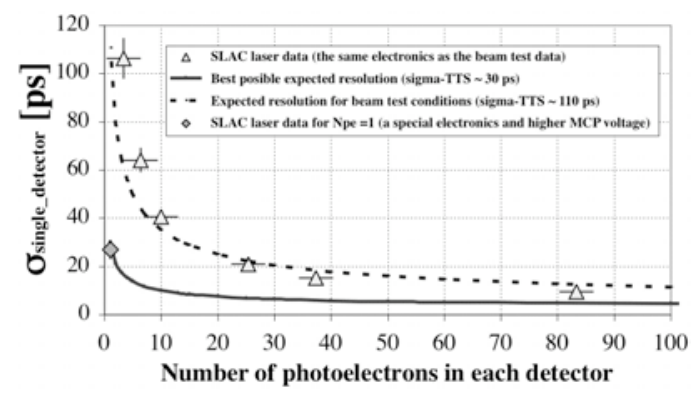

Fig. 5 Measured timing resolution as a function of number of photoelectrons $\left(N_{\mathrm{pe}}\right)$ for (a) the electronics intended for the beam test ${ }^{6}($ not sensitive to single $\mathrm{Npe}=1$, but linear for $\mathrm{Npe}=30-50$ ), and (b) the electronics sensitive to single pe [1]. Dashed curve assumes ${ }^{1} \sigma_{\mathrm{TTS}} \sim 110$, solid curve assumes ${ }^{2} \sigma_{\mathrm{TTS}}=27 \mathrm{ps}$.

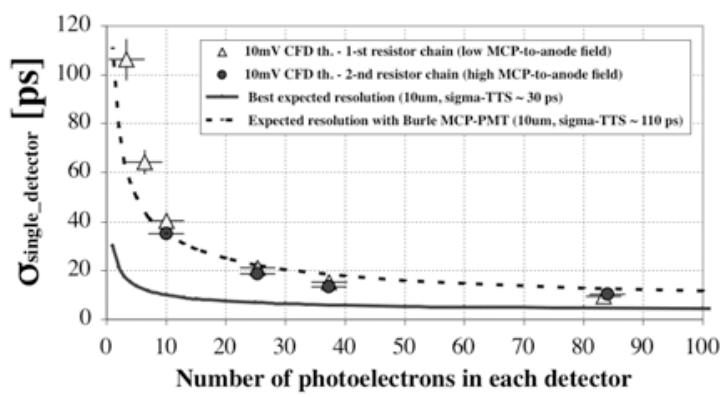

Fig. 6 Timing resolution as a function of number of photoelectrons $\left(\mathrm{N}_{\mathrm{pe}}\right)$ for two resistor chains, providing two MCP-to-anode electric fields: $\sim 400 \mathrm{~V} / \mathrm{cm}$ and $\sim 940 \mathrm{~V} / \mathrm{cm}$. The theoretical curves are the same as in Fig.5.

We tried to improve the resolution by reducing the MCP rise time. This can be done, for example, by doubling the MCP-to-anode electric field [5] by modifying the MCP resistor chain. ${ }^{10}$ Fig. 6 shows that the results are slightly better at higher electric field.

For a red laser and Bialkali photocathode, the emitted photoelectrons are practically at rest and the MCP rise time does not change as a function of the cathode-to-MCP electric field [5]; therefore we did not vary this field.

For any timing method there is some time-walk when one varies the number of photoelectrons $\left(\mathrm{N}_{\mathrm{pe}}\right)$ [6], and this effect has to be corrected off-line to achieve the best possible timing resolution. The amplitude correction is not entirely easy, as the MCP pulses are very fast. Fig. 7 shows the time-walk for the measurements shown in Fig. 6 using Ortec $9327 \mathrm{CFD}$; it is small for $20 \leq \mathrm{N}_{\mathrm{pe}} \leq 50$, and it is also smaller for the 2nd resistor chain, which means the time-walk is smaller for smaller MCP rise time.

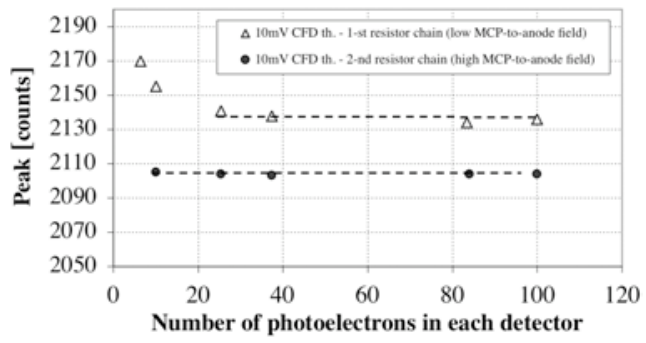

Fig. 7 CFD time-walk as one varies the number of photoelectrons in the measurements in Fig. 6 (3.19 ps/count).

\footnotetext{
10 Another example: smaller MCP hole dia., smaller rise time [6].
} 


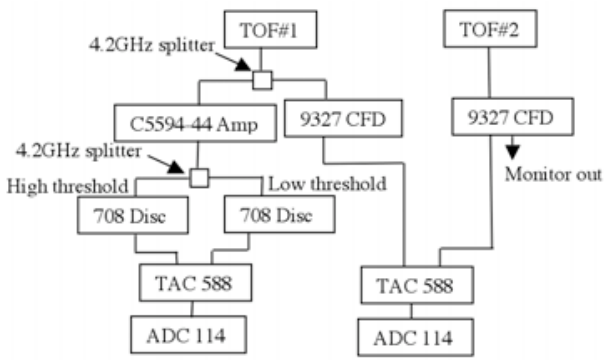

Fig. 8 Schematic of the circuit added to the TOF1 counter, based on Ortec electronics, ${ }^{6}$ which is capable of correcting the pulse height variation.

To see if we can improve the above results further by additional pulse height correction, especially for small $\mathrm{N}_{\mathrm{pe}}$ values, we have added double-threshold timing into the circuit - see Fig. 8. Although one can nicely correct the pulse height variation using this method, in the final analysis, the results are not better compared to the method using the circuit on Fig.3, which indicates that the CFD timing handles a small level of pulse height variation rather well.

\section{Lessons from the test beam}

We have tested the tandem of two detectors in a $10 \mathrm{GeV} / \mathrm{c}$ electron test beam at SLAC. The best expected $\mathrm{N}_{\mathrm{pe}}$ is $\sim 30-40$ from a $10 \mathrm{~mm}$-long quartz radiator and the Burle Bialkali photocathode data for our two tubes. In addition, the aluminium coating of the quartz rods was as not uniform. As can be seen in Fig. 7, time-walk correction is necessary for $\mathrm{N}_{\mathrm{pe}}<20$. There was no external pulse height measurement implemented in this particular beam test to correct the time-walk. During the test we used the 1st resistor chain (triangles in Fig. 7). If we had used the 2 nd resistor chain the time-walk would be smaller. In addition to the time-walk contribution, the expected resolution is worse for smaller $\mathrm{N}_{\mathrm{pe}}-$ see Figs. 5 and 6. As a result of these contributions, our initial test beam result resulted only in $\sigma_{\text {Single_detector }} \sim 23$ ps. ${ }^{11}$

Possible ways to improve the result in future are: (a) implement a pulse height measurement on fast MCPPMT pulses, (b) ensure that $\mathrm{N}_{\mathrm{pe}}>25$, (c) run with highest possible MCP-to-cathode and anode-to-MCP electric fields, (d) small MCP-to-cathode and anode-to-MCP gaps, and (e) have smallest possible MCP holes, or (f) for some low rate applications use timing method described in Ref.1.

\section{Acknowledgments}

We would like to thank M. McCulloch for help to prepare the detector setup. We thank H. Frisch, G. Varner, K. Byrum, G. Drake and F. Tang for sharing ideas and resources, and P. Hink of Burle/Photonis for useful advice and help.

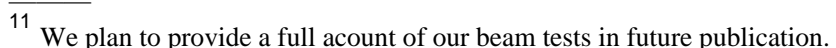




\section{References}

[1] J. Va'vra, J.F. Benitez, D.W.G.S. Leith, G. Mazaheri, B. Ratcliff and J. Schwiening, "A 30 ps timing resolution for single photons with multi-pixel Burle MCP-PMT,” Nucl. Instr.\&Meth., A572(2007)459-462.

[2] T.Ohshima, K.Inami, N.Kishimoto, M.Nagamine, Nagoya University, Japan. Talk at "International Symposium on the Development of Detectors", April 2006, SLAC., and T.Ohshima, "The Pico-Sec Timing Workshop," 18 Nov 2005, U. of Chicago, http://hep.uchicago.edu/psecl, and Nucl. Instr.\&Meth., A528(2004)763-775.

[3] Super-B CDR report, INFN/AE-07/2, SLAC-856, LAL07-15, March 2007.

[4] B. Ratcliff, invited talk at this workshop.

[5] J. Milnes and J. Howorth, "Picosecond Time Response Characteristics of Micro-channel Plate PMT Detectors," Photek Ltd. internal publication, September 1, 2004, james.milnes@photek.co.uk

[6] J. Va'vra, J. Benitez, D.W.G.S. Leith, G. Mazaheri, B. Ratcliff, J. Schwiening, and K. Suzuki, "The Focusing DIRC - the first RICH detector to correct the chromatic error by timing, and the development of a new TOF detector concept", March, 2006, SLAC-PUB12803. 